\title{
Central nervous system imaging and congenital melanocytic naevi
}

\author{
V A Kinsler, S E Aylett, S C Coley, W K Chong, D J Atherton
}

\begin{abstract}
Aim-To establish the prevalence of central nervous system (CNS) abnormalities on magnetic resonance imaging (MRI) in a population of children with congenital melanocytic naevi (CMN) over the head and/or spine, and to compare this with clinical findings.

Methods-Forty three patients identified from outpatient clinics underwent MRI of the brain and/or spine. These were reported by a paediatric radiologist and findings compared with the clinical picture.

Results-Nine patients had abnormal clinical neurology, seven had abnormal findings on MRI, and six had both abnormal clinical and radiological findings. Only three of the abnormal MRIs showed features of intracranial melanosis. Three others showed structural brain abnormalities: one choroid plexus papilloma, one cerebellar astrocytoma, and one posterior fossa arachnoid cyst; the first two of these have not previously been described in association with CMN. The last abnormal MRI showed equivocal changes requiring reimaging.

Conclusions-The prevalence of radiological CNS abnormality in this group of children was $7 / 43$. Six of these developed abnormal clinical neurological signs within the first 18 months of life, but two did not do so until after the MRI. Two of the CNS lesions were operable; for this reason we support the routine use of early MRI in this group.

(Arch Dis Child 2001;84:152-155)
\end{abstract}

Dermatology, Great

Ormond Street

Hospital for Children

NHS Trust, Great

Ormond Street,

London WC1N 3JH,

UK

V A Kinsler

D J Atherton

Neurosciences Unit, Great Ormond Street Hospital for Children NHS Trust

S E Aylett

Department of Radiology, Great Ormond Street Hospital for Children NHS Trust,

S C Coley

W K Chong

Correspondence to: Dr Atherton

Accepted 13 July 2000 nucleus, cerebellar hemispheres, pons, thalamus, and amygdala. Neurological symptoms may occur; these may be caused by abnormalithe brain. In NCM, thick sheets of melanocytes are found in the pia and arachnoid, most commonly over the base of the brain, and in the parenchyma of the basal ganglia, dentate ties of circulation, resorption of cerebrospinal fluid, local pressure effects, or malignant transformation. In the past, intracranial melanosis (ICM) would only become apparent if the patient developed neurological symptoms, and the diagnosis could not usually be made in life. At that time, the condition was perceived as almost invariably lethal.

The advent of non-invasive imaging techniques, such as magnetic resonance imaging (MRI), has begun to change our perception of CNS abnormalities in patients with CMN. Firstly, it has become apparent that CMN may be associated with a variety of non-melanocytic anomalies of the CNS, including DandyWalker malformation, ${ }^{1}$ encephalocoele, ${ }^{2}$ inferior vermian hypoplasia, ${ }^{3}$ multiple meningiomas, ${ }^{4}$ partial agenesis of the right parietal lobe, ${ }^{5}$ middle cranial fossa arachnoid cyst, and Chiari type I malformation. ${ }^{6}$ Secondly, it now seems clear that there are individuals with $\mathrm{CMN}$ who have asymptomatic ICM.${ }^{6}{ }^{7}$ Neither the frequency nor the natural history of asymptomatic ICM have been established; neurologically symptomatic ICM (defined as abnormal neurological symptoms and/or signs) is considered to carry a very high mortality rate. $^{78}$

Two studies concerning this issue have produced disparate results. In the first, MRI was performed on 20 neurologically asymptomatic children with large or multiple CMN, who were found to have a high prevalence of MRI abnormalities (45\%). ${ }^{6}$ The principal cause of an abnormal MRI was asymptomatic ICM $(33 \%)$. In the second, 13 patients had MRI; all were thought to be neurologically asymptomatic, but 11 were subsequently found to have neurological signs on examination. ${ }^{9}$ None of these patients had ICM on MRI, although six had anatomical abnormalities of the CNS. This study implies a low rate of asymptomatic ICM.

The availability of MRI has therefore raised several interesting questions about CNS involvement in patients with CMN. Firstly, what is the prevalence of ICM and other CNS abnormalities in this group of patients? Secondly, can one predict which patients will have such abnormalities on the basis of their clinical picture (cutaneous lesions and neurology)? Thirdly, what is the outlook for patients with symptomatic or asymptomatic ICM, if the latter exists? This study has been undertaken to address the first two of these questions, and to initiate investigation of the third. From the clinical viewpoint, the principal aim of the study has been to provide data that indicate which patients with CMN should be imaged, 


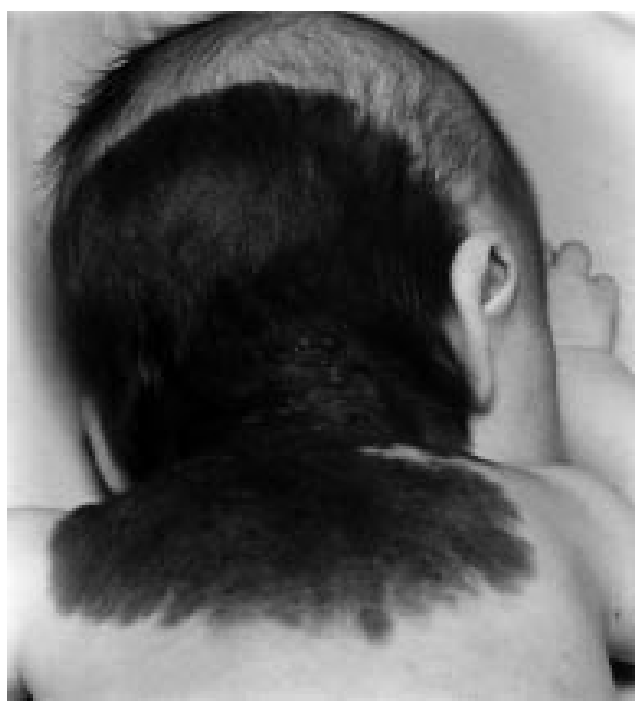

Figure 1 Congenital melanocytic naevus on upper back and occiput in an infant.

and what the imaging findings mean for the individual.

\section{Patients and methods}

There is evidence that symptomatic intracranial abnormalities are more often associated with CMN located on the head or over the spine. ${ }^{78}$ For this reason, we offered MRI of the brain and/or spine to children with $\mathrm{CMN}$ of diameter greater than $2 \mathrm{~cm}$ on the head or overlying the spine (fig 1). The arbitrary figure of $2 \mathrm{~cm}$ was chosen for practical reasons to exclude the very common smaller naevi, but to include all others as there are no data which establish that only giant CMN are associated with neurological abnormalities. For the same reason, we did not attempt to assess the future size of the naevi based on surface area projections. The $2 \mathrm{~cm}$ cut off also means that most children with multiple naevi will be included.

All children were attending the paediatric dermatology clinic either at Great Ormond Street Hospital for Children or St John's Institute of Dermatology. Children were not recruited from a neurological or neurosurgical service, and there is therefore a slight possibility of bias in the patient selection. A paediatrician took a neurological history and undertook a neurological examination on each child as part of their clinical assessment. Abnormal clinical neurology was defined as any neurological symptom or sign or developmental delay. Age of onset of abnormal neurology was defined as the age at which abnormal neurological symptoms or signs were first noted in the history or examination. It is therefore possible that some of the neurological abnormalities were present before the times recorded, but had not been detected.

The clinical notes and MRI scans of 51 children were examined. Clinical and imaging data were available for 43 children (20 boys). Mean age at MRI was 4.35 years (range $0.3-16.9$ years), with a median of 2.6 years. Table 1 shows the location and size of the largest CMN in each individual. Thirty four children were documented as having satellite lesions, five did not have satellites, and there was no information regarding satellite lesions at the time of imaging in the remaining four.

MRI was performed at $1.5 \mathrm{~T}$ with $\mathrm{T} 1$ and $\mathrm{T} 2$ weighted imaging. MRI was chosen because the paramagnetic effect of melanin leads to a decrease in both $\mathrm{T} 1$ and $\mathrm{T} 2$ relaxation times, with highly characteristic appearances on imaging. ${ }^{10}$ Sixteen children had brain scans alone, four had spinal imaging alone, and 23 had both. All children with abnormal clinical neurology had brain scans. Six children received intravenous gadolinium contrast material. The scans were interpreted by a paediatric neuroradiologist, who was unaware of the clinical findings, other than that all the children had $C M N$.

\section{Results}

Nine children had abnormal neurological symptoms or signs. MRI abnormalities were found in seven, and six had both abnormal scans and neurology. Table 2 shows the clinical features and MRI findings. Of the seven children with abnormal scans, three had intracranial melanosis. The non-melanocytic CNS lesions were a benign fibrotic disorganised choroid plexus lesion which was resected at age 2 years, a juvenile cerebellar astrocytoma which was partially resected at age 2 years, and communicating hydrocephalus with no obvious structural cause. The seventh abnormal scan (at age 2.0 years) showed changes which were almost certainly a result of immature myelination; this patient will be imaged after an interval to clarify the situation. To our knowledge, the benign non-melanotic choroid plexus papilloma and the juvenile cerebellar astrocytoma have not previously been described in association with $\mathrm{CMN}$.

All except one of the seven children with an abnormal MRI developed abnormal neurological symptoms or signs in the first 18 months of life (see tables 3 and 4), though because the scans were undertaken early in some of the children, two of these did not do so until after the MRI. Only one patient had an abnormal MRI scan without clinical neurological problems; this patient had changes suggestive of immature myelination. Three patients had neurological symptoms or signs with normal MRI scans.

\section{Discussion}

The prevalence of CNS abnormalities on MRI in our patients is $7 / 43$. Three had typical scan findings of ICM; the other four had nonmelanocytic lesions. Of these four, one had posterior fossa abnormalities of a type that has previously been reported in association with CMN, one had an abnormality of doubtful significance (immature myelination), and two had lesions that have never been described in association with $\mathrm{CMN}$. A very high proportion of children (6/7) with abnormal MRI also had clinical neurological symptoms and/or signs within the first 18 months of life, giving a prevalence of symptomatic neurological abnormality of $14 \%$. While it is possible that our 


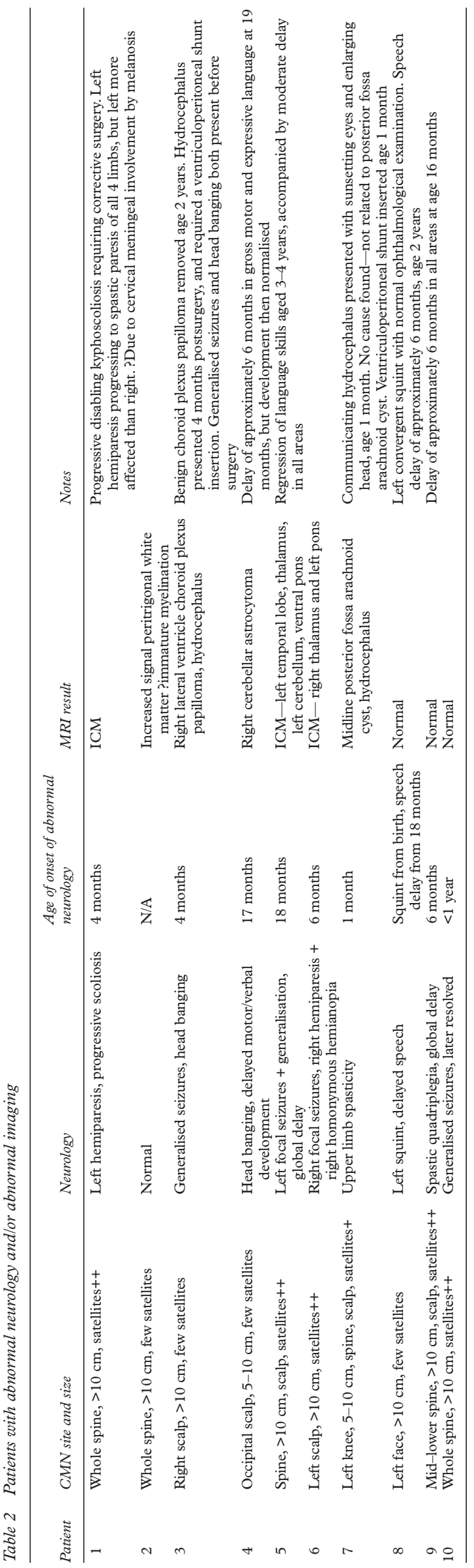

Table 3 Association between clinical neurology and results of scans

\begin{tabular}{lllr}
\hline & $\begin{array}{l}\text { Normal } \\
\text { neurology }\end{array}$ & $\begin{array}{l}\text { Abnormal } \\
\text { neurology }\end{array}$ & Total \\
\hline Normal MRI & 33 & 3 & 36 \\
Abnormal MRI & 1 & 6 & 7 \\
Total & 34 & 9 & 43 \\
\hline
\end{tabular}

Table 4 Abnormal results classified by site and size of the largest $C M N$

\begin{tabular}{llll}
\hline & $\begin{array}{l}\text { Patient } \\
\text { numbers }\end{array}$ & $\begin{array}{l}\text { Abnormal } \\
\text { neurology }\end{array}$ & $\begin{array}{l}\text { Abnormal } \\
\text { scan }\end{array}$ \\
\hline CMN site & & & \\
Head & 10 & 3 & 2 \\
Head + spine & 16 & 4 & 3 \\
Spine & 17 & 2 & 2 \\
Total & 43 & 9 & 7 \\
$C M N$ size & & & \\
$<5 \mathrm{~cm}$ & 1 & 0 & 0 \\
$5-10 \mathrm{~cm}$ & 10 & 2 & 2 \\
$>10 \mathrm{~cm}$ & 32 & 7 & 5 \\
Total & 43 & 9 & 7 \\
\hline
\end{tabular}

patient base may be biased towards the more severe end of the spectrum of CMN, this prevalence is comparable to that reported by DeDavid et al in their patients with posterior axial CMN and symptomatic CNS involvement $(13 \%){ }^{7}$

The possibility that some patients might have asymptomatic ICM was originally raised when it was found incidentally during postmortem examinations of patients with CMN. ${ }^{11}$ Frieden et al used MRI to pursue this possibility, and found a surprisingly high frequency of MRI abnormalities in neurologically asymptomatic patients (9/20). ${ }^{6}$ A high proportion of those with abnormal MRI had ICM (6/9); the overall prevalence of ICM in this group of asymptomatic patients was $6 / 20$. This appears to contrast sharply with our finding of only one neurologically normal patient with an abnormal MRI. This difference may be a result of the earlier age at which scans were undertaken; the median age of the 20 children in the study by Frieden et al was 2 months (compared to 2.6 years in our study). The scans may therefore have been undertaken before the onset of clinically detectable neurological abnormalities. In an earlier report, Kadonaga and Frieden ${ }^{8}$ noted that while more than half of the clinical neurological abnormalities became detectable in the first two years of life, others did not do so until the second and third decades.

Ruiz-Maldonado et al found a low prevalence of asymptomatic abnormalities on MRI in their study of 13 people (11 of whom were children; median age 11.6 years) with $\mathrm{CMN}$ over the head and or spine. ${ }^{9}$ However, this study involved relatively small numbers, particularly of asymptomatic patients (only two of 13). Interestingly none of the abnormal MRIs in their series (six of 13) showed ICM, but again this may be a result of small patient numbers.

The prognosis for children with ICM remains unclear. In this study, all children in whom ICM was detected in the first 18 months of life already had neurological abnormalities. 
It was previously argued that clinicians would be less likely to be aggressive in the management of the cutaneous lesions if there was known involvement of the CNS, but this was when CNS involvement was thought always to carry a poor prognosis. It now appears likely that progressive neurological deterioration is not invariable in these cases, but long periods of observation will be required to clarify the situation. There is very little evidence at this stage to suggest that early detection of ICM alters prognosis.

Relating the MRI findings to the clinical characteristics of the patients with CMN is hampered by the relatively small numbers of patients. For example, there is only one patient whose largest CMN is less than $5 \mathrm{~cm}$ in diameter. There is however no suggestion that the prevalence of abnormality in the size groups $5-10 \mathrm{~cm}$ and $>10 \mathrm{~cm}$ is strikingly different. This may be a result of the arbitrary nature of a division at $10 \mathrm{~cm}$. This challenges the previous supposition that neurological abnormality is only associated with giant or multiple naevi.

It is interesting to speculate on the different types of intracranial lesion and possible associations with different types of CMN. From our small sample, there is a suggestion that large scalp lesions may be associated with directly underlying non-melanocytic CNS malformation (table 2 ; patients 3 and 4). It may be relevant that these two patients had very few satellite lesions. On the other hand, all three of our patients with intracranial melanosis had large numbers of satellite lesions.

Because only one patient had an abnormal scan in the absence of abnormal neurology in the first 18 months of life, one could argue that MRI should not be undertaken routinely in clinically normal children with CMN. This view would appear to be strengthened by the fact that the abnormality found on this patient's MRI was inconclusive, and a second scan will be required after an interval. It is recognised that MRI examinations are more difficult to acquire and more difficult to interpret under 1 year of age. However, in our group of nine children with an abnormal MRI, there were two with surgically treatable lesions (a benign non-melanocytic choroid plexus papilloma and a juvenile cerebellar astrocytoma) which might have been detected even more promptly by an earlier scan, and treated prior to the onset of seizures. Therefore, we support the use of early routine MRI. The principal reason for such scans is the detection of operable lesions. These will almost invariably be non-melanocytic lesions, as ICM is not currently amenable to surgical treatment.

In conclusion, we would recommend that all children conforming to the selection criteria used in this study - that is, a CMN greater than $2 \mathrm{~cm}$ in diameter on the head or over the spine, should have a careful neurological and developmental assessment by a paediatrician at presentation, and should continue to be followed closely during at least the first two years. We support the use of early MRI, but the justification is the detection of operable presymptomatic non-melanocytic lesions-a type of lesion that appears to be more common in patients with CMN than in the general population. Detailed clinical and radiological follow up data on these and other groups of similar patients are required to elucidate the natural history of asymptomatic radiological CNS abnormalities, and to make recommendations about long term follow up.

This work was undertaken by Great Ormond Street Hospital for Children NHS Trust which received a proportion of its funding from the NHS executive. The views expressed in this publication are those of the authors and are not necessaily those of the NHS executive. Since submitting this paper, patient 2 in of the NHS executive. Since submitting this paper, patient 2 in
table 2 has had a repeat MRI of the head, which is entirely table 2 has had a repeat MRI of the head, which is entirely
normal. This confirms that the original abnormalities were probably due to immature myelination.

1 Kadonaga JN, Barkovich AJ, Edwards MSB, Frieden IJ. Neurocutaneous melanosis in association with the DandyWalker complex. Pediatr Dermatol 1992;9:37-43.

2 Shermak MA, Perlman EJ, Carson BS, Dufresne CR. Giant congenital nevocellular nevus overlying an encephalocele. $\mathcal{f}$
cont Craniofac Surg 1996;7:376-83.

3 Ko SF, Wang HS, Lui TN, Ng SH, Ho YS, Tsai CC. Neurocutaneous melanosis associated with inferior vermian hypoplasia: MR findings. I Comput Assist Tomogr 1993;17: 691-5.

4 Hara M, Okabe T, Inafuku T, Hamaguchi K, Yamada F. A case of giant pigmented nevus with multiple meningiomas. Rinsho Shinkeigaku 1992;32:457-60.

5 Garcia Perres JJ, Ramos Lizana J, Roche Herrero C, Carrascosa Romero C, Pascual Castroviejo I. Neurocutaneous melanosis associated with partial agenesis of the right parietal lobe. An Esp Pediatr 1992;36:239-41.

6 Frieden IJ, Williams ML, Barkovich AJ. Giant congenital melanocytic nevi: brain magnetic resonance findings in melanocytic nevi: brain magnetic resonance findings in neurologically

7 DeDavid M, Orlow S, Provost $\mathrm{N}$, et al. Neurocutaneous melanosis: clinical features of large congenital melanocytic nevi in patients with manifest central nervous system melanosis. $\mathcal{F}$ Am Acad Dermatol 1996;35:529-38.

8 Kadonaga JN, Frieden IJ. Neurocutaneous melanosis: definition and review of the literature. $\mathcal{F}$ Am Acad Dermatol 1991;24:747-55

9 Ruiz-Maldonado R, del Rosario Barona-Mazuera M, Hidalgo-Galvan LR, et al. Giant congenital melanocytic nevi, neurocutaneous melanosis and neurological alterations. Dermatology 1997;195:125-8.

10 Barkovich AJ, Frieden IJ, Williams ML. MR of neurocutaneous melanosis. Am f Neuroradiol 1994;15:859-67.

11 Slaughter JC, Hardman JM, Kempe LG, et al. Neurocutaneous melanosis and leptomeningeal melanomatosis in children. Arch Pathol 1969;88:298-304. 